SLAC-PUB-8103

March 1999

\title{
THE TRANSVERSE LONG-RANGE WAKEFIELD IN RDDS1 FOR THE JLC/NLC X-BAND LINACS
}

\author{
R.M. Jones ${ }^{\dagger}$, N.M. Kroll ${ }^{\dagger \dagger}$, R.H. Miller ${ }^{\dagger}$, T. Higo ${ }^{\ddagger}$, Z. Li $^{\dagger}$, R.D. Ruth ${ }^{\dagger}$ and J. W. Wang ${ }^{\dagger}$ Stanford \\ $\dagger$ Stanford Linear Accelerator Center, M/S 26, P.O Box 4349, Stanford, CA 94309, USA \\ ¥University of California, San Diego, La Jolla, CA 92093-0319. \\ $¥$ ¥EK, National Laboratory for High Energy Physics, 1-1 Oho, Tsukuba-shi, \\ Ibaragi-ken 305, Japan
}

\begin{abstract}
The re-designed RDDS (Rounded Damped Detuned Structure) consists of 206 cells with a rounded cell profile formed by a number of circular arcs and a straight section. In the previous analyses [1] of the present structure all cells have been assumed to be coupled to the manifold via slots cut into the cells and, a perfect match to the HOM (Higher Order Mode) couplers has been used. In the structure being fabricated, four cells on either end of the structure are decoupled to accommodate the HOM couplers and, the HOM couplers have finite, frequency dependent, reflection coefficients. The wakefield is calculated incorporating both effects
\end{abstract}

Paper presented at the 1999 Particle Accelerator Conference (PAC99)

New York City, New York, USA

March 29-April 2, 1999

This work is supported by Department of Energy grant numbers DE-AC03-76SF00515 ${ }^{\dagger}$ and DE-FG03-93ER40759 . $^{\ddagger}$ 


\title{
THE TRANSVERSE LONG-RANGE WAKEFIELD IN RDDS1 FOR THE JLC/NLC X-BAND LINACS
}

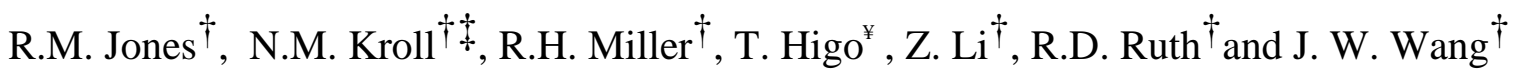 \\ $\dagger$ Stanford Linear Accelerator Center, M/S 26, P.O Box 4349, Stanford, CA 94309, USA \\ ¥University of California, San Diego, La Jolla, CA 92093-0319. \\ $¥$ ¥EK, Natl. Lab. for High Energy Physics, 1-1 Oho, Tsukuba-shi, \\ Ibaragi-ken 305, Japan
}

\section{Abstract}

The re-designed RDDS (Rounded Damped Detuned Structure) consists of 206 cells with a rounded cell profile formed by a number of circular arcs and a straight section. In the previous analyses [1] of the present structure all cells have been assumed to be coupled to the manifold via slots cut into the cells and, a perfect match to the HOM (Higher Order Mode) couplers has been used. In the structure being fabricated, four cells on either end of the structure are decoupled to accommodate the HOM couplers and, the HOM couplers have finite, frequency dependent, reflection coefficients. The wakefield is calculated incorporating both effects.

\section{INTRODUCTION}

In order to achieve high luminosity beams at the collision point in a linear collider scheme it is required to accelerate multiple bunches of low emittance particles. However, the first bunch in a train, will excite a transverse

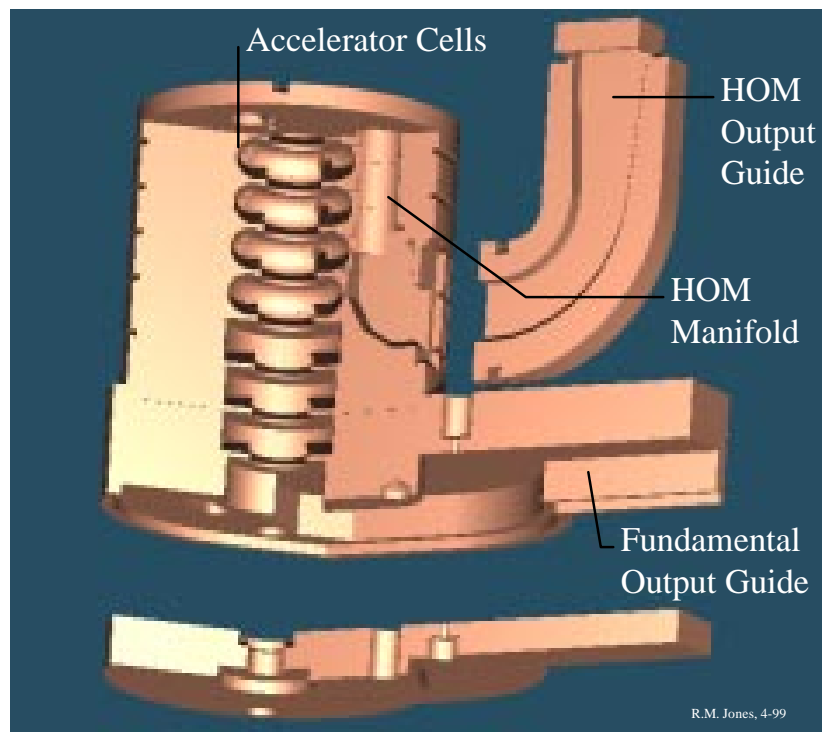

Figure 1: CAD exploded view of RDDS. The curvature of the cells and the HOM couplers are illustrated.

wakefield, which arises either by a slight transverse misalignment in the structure, or some jitter induced beam offset. This wakefield will kick successive bunches off axis and they will also generate wakefields themselves. If this effect is not reduced then the emittance of the beam will be diluted and a BBU (Beam Break Up) instabilty will develop. The main component of the modes is dipole in character. In the KEK/SLAC collaboration we have developed a scheme to damp these modes, which relies on Gaussian detuning the modes excited by the beam such that they do not add up in a coherent fashion, together with moderate damping of the long range wakes. The RDDS is illustrated in Fig. 1. Damping is required in addition to detuning because eventually the finite number of modes will partially recohere and lead to an unacceptably high wakefield. The long-range wakefield is carefully coupled out at each cell to four manifolds which lie parallel to the axis of the accelerator.

There have been three DDS, two of which, DDS 1 and 3 have been measured in the SLC using the ASSET facility [2]. The present design, RDDS1, has a $11.25 \%$ frequency bandwidth or about 4.75 in units of $\sigma$. This design has been carefully optimized to maximize the shunt impedance and to minimize the long range transverse wakefields. In the process of designing the structure it was necessary to decouple cells and it was found that, there will not in practice be a perfect match of the HOM load. The next section discusses the design of the load and in section 3, the influence of decoupling cells on thec wakefield is described.

\section{MANIFOLD TERMINATIONS}

The HOM terminations for the DDS are surprisingly critical: a VSWR as small as 1.05 on the loads at the downbeam end of the structure causes a noticeable increase in the wakefield amplitudes. However, our equivalent circuit model shows that the power flow in the manifold is predominantly in the direction of the beam, so that reflections at the upbeam end are of little consequence. In order to avoid the possibility of stray electrons charging the lossy ceramic in the loads and also the possibility of ceramic fragments falling into the accelerator structure, we have chosen to have the loads external to the accelerator structure. The power is brought out in rectangular double-mitered bends which have an excellent match over the $11 \%$ bandwidth of interest. The match from the round manifolds which are periodically loaded by coupling apertures into each cell (except the final 4) into the smooth rectangular guide in a short distance (order of $10 \mathrm{~mm}$ ) proved to be more difficult. For both the numerical modeling and the experimental cold test and optimization measurement the same approach 

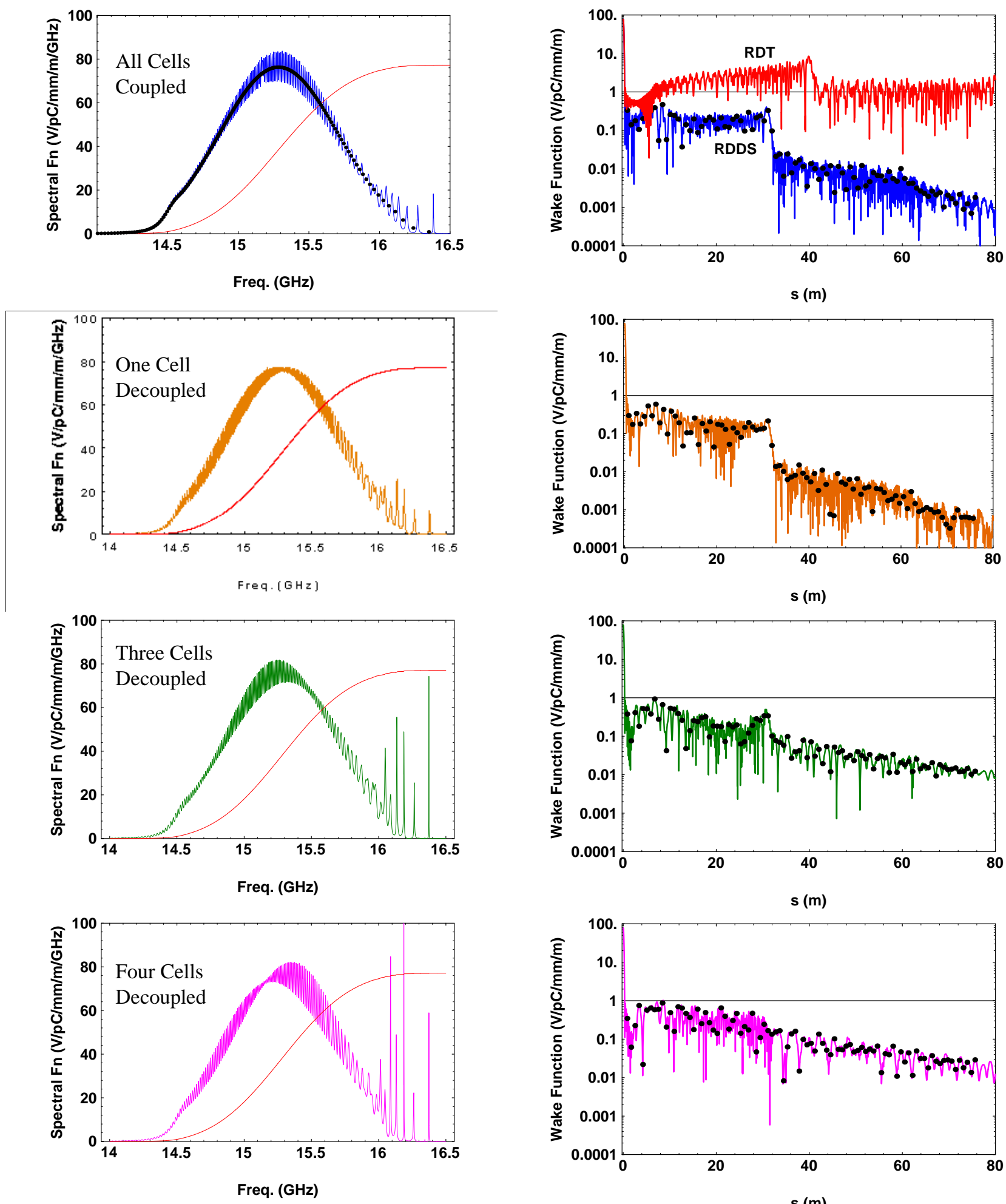

Figure 2: Uppermost is the spectral function for all cells coupled (where the dots indicate the smoothed undamped spectral function). The subsequent curves are computed for 1 cell decoupled, followed by three, and four cells (lowermost) decoupled from both the upstream and downstream end of the RDDS

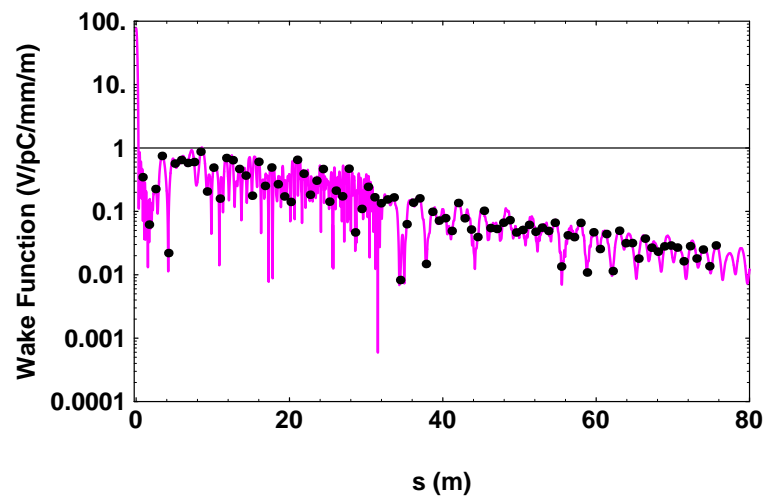

Figure 3: Uppermost is shown a comparison of the RDDS versus the RDT (Rounded Detuned, see main text) wake function for all cells coupled. The subsequent curves are computed for 1 cell decoupled, three, and finally 4 cells (lowermost) decoupled from both the upstream and downstream end of the RDDS. 


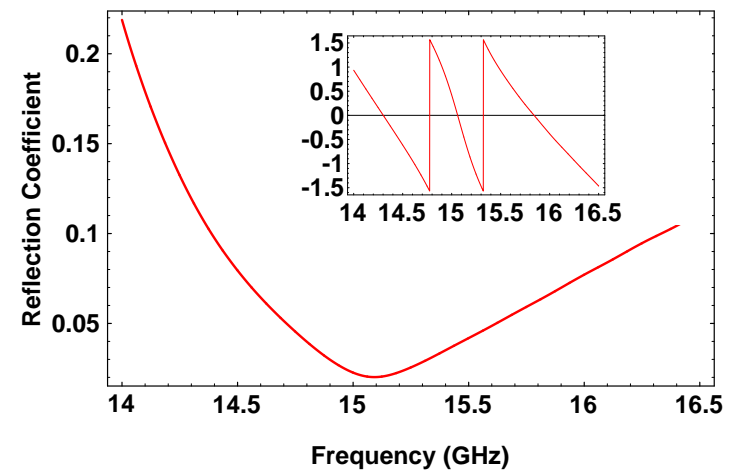

Figure 4: Magnitude of the reflection coefficient of the HOM couplers (shown inset is the corresponding phase)

was taken: two higher order mode couplers are put back and the distance between them was varied by integer numbers times the periodic length of the structure. So the rf power traveled from a source through a single mode rectangular waveguide, through the double mitered bend, through the transition from rectangular guide to the round $\mathrm{TE}_{11}$ manifold periodically loaded by slots into the accelerator cells every $8.75 \mathrm{~mm}$, up to about 5 periods, through another transition back to a smooth rectangular guide, and finally through the second double mitered bend to an output single mode rectangular guide to a matched load. If the system is lossless, as the number of periods between the input transition and the output transition is varied, the input impedance always lies on a circle in the complex plane. If the input and output transitions are identical, the circle passes through the origin so, in principle, two measurements determine the circle. For both the numerical simulation and the cold tests 3 or 4 different lengths are used, and for each length the impedance is measured as a function of frequency over the band from 14.0 to $16.5 \mathrm{GHz}$. The design was optimized using MAFIA in time domain driving the system with a pulse short enough to contain the required bandwidth. The forward and reflected waves were Fourier analyzed and the reflection coefficient calculated as a function of frequency. The reflection coefficient for a single HOM coupler calculated from the numerical simulation is shown in Fig 4. A bolt-together cold test model which permits changing the length between the transitions in period steps has been built and cold testing has begun.

\section{DECOUPLED CELLS}

The reason we are required to minimize the wakefield is that a cumulative BBU instability may develop if the wakefield couples resonantly to multiple bunches and kicks them off axis. Provided the wakefield is sufficiently small the BBU instability will not develop. In Fig 1, uppermost, the spectral function is computed for all cells coupled to the manifold and including the effect of the mismatch of the frequency dependent HOM coupler (shown in Fig 3). The oscillation imposed on the spectral function results from the imperfect match of the HOM coupler. The resulting wakefield is shown uppermost in Fig. 3. There is some degradation in the reduction in wakefield in the range 5 to $30 \mathrm{~m}$ or so. Also indicated in Fig. 2 is the wakefield that would result if none of the cells were coupled to the manifold, i.e., if a purely DT (detuned structure) The wakefield resulting from the DT recoheres and for this reason additional damping is required.

The wakefield when all cells are coupled to the manifold, at the design value of the bunch spacing is sufficiently small to prevent BBU occurring. However, it has been found that the sum wake even for all cells coupled is quite sensitive to bunch spacing and this situation may give rise to a significant growth in the emittance. Including a perfect match to the HOM improves the wakefield and alleviates BBU. However, in practice mechanical design considerations require that a number of cells be decoupled. The resulting spectral function is shown in Fig. 1 (third from the top). Inspection of the spectral function in the high frequency region reveals a number of high Q modes have resulted from the decoupling. These high $\mathrm{Q}$ modes lead to an increase in the wakefield (shown by the third curve in Fig 2) in the region 4-8 meters and in the region immediately after the recoherence peak (situated at approximately $30 \mathrm{~m}$ ).

Decoupling cells enhances the wakefield to the extent that a single mode, or small number of modes, drives the bunches off axis and this results in BBU at the designed bunch spacing. Further, the difference between the wakefield achieved with 3 and 4 cells decoupled (lowermost in Fig 2) is seen to be quite small. And hence, to further ease the constraints on the method of minimizing the reflection coefficient of the transition between circular manifold and rectangular manifold (and on mechanical constraints imposed on the geometry) we finally decided upon a RDDS with 4 cells decoupled from the manifold.

\section{RDDS EXPERIMENTS ENVISAGED}

In the forthcoming ASSET experiment planned to measure the wakefield in RDDS 1 we will carefully design the horizontal manifold, such that only one cell is decoupled. This will allow us to compare the wakefields as a function of cell decoupling. Additionally, it may be possible to utilize the NLCTA to investigate BBU.

\section{ACKNOWLEDGMENTS}

This work is supported by Dept. of Energy grant numbers DE-AC03-76SF00515 ${ }^{\dagger}$ and DE-FG03-93ER40759 $9^{\ddagger}$.

\section{REFERENCES}

[1] R.M.Jones et al, Linac98 Conf., (SLAC-PUB 7934)

[2] C. Adolphsen et al, this Conf., FRA40 